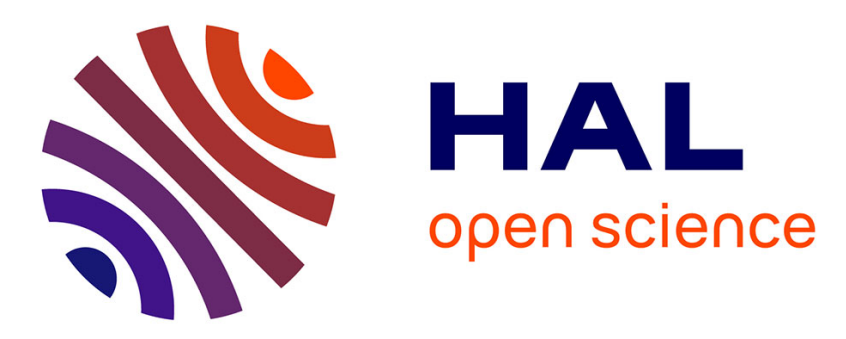

\title{
La formulation du modèle keynésien et le choix de l'unité de mesure
}

\author{
Alain Béraud
}

\section{To cite this version:}

Alain Béraud. La formulation du modèle keynésien et le choix de l'unité de mesure. Recherches Economiques de Louvain - Louvain economic review, 2014, 80 (3), pp.21-50. 10.3917/rel.803.0021. halshs-01065712

\section{HAL Id: halshs-01065712 https://shs.hal.science/halshs-01065712}

Submitted on 18 Sep 2014

HAL is a multi-disciplinary open access archive for the deposit and dissemination of scientific research documents, whether they are published or not. The documents may come from teaching and research institutions in France or abroad, or from public or private research centers.
L'archive ouverte pluridisciplinaire HAL, est destinée au dépôt et à la diffusion de documents scientifiques de niveau recherche, publiés ou non, émanant des établissements d'enseignement et de recherche français ou étrangers, des laboratoires publics ou privés. 


\title{
LA FORMULATION DU MODELE KEYNESIEN ET LE CHOIX DE L'UNITE DE MESURE
}

\author{
Alain Béraud ${ }^{1}$
}

Résumé : Cet article analyse la façon dont Keynes et les keynésiens posèrent la question du choix de l'unité de mesure des agrégats macroéconomiques. Soulignant les rapports étroits qui existent entre les cours que Keynes professa entre 1933 et 1935 et les diverses versions du modèle IS-LM, il montre que les problèmes naissent de la façon dont Keynes avait abordé ce problème. Raisonner sur la valeur monétaire des agrégats conduit à dichotomiser le modèle et ne permet pas d'analyser correctement l'interdépendance entre le marché du travail d'une part et les marchés des biens et de la monnaie d'autre part. Dans le modèle ainsi formulé, il est impossible d'étudier de façon rigoureuse les effets d'une variation du salaire monétaire et l'on est conduit à penser, à tort, qu'il existe nécessairement un équilibre de plein emploi si le taux de salaire monétaire est flexible.

Abstract: This contribution analyzes how Keynes and the Keynesians asked the question of the choice of the unit of measure of the macroeconomic aggregates. Underlining the narrow relationships which exist between the lectures Keynes professed between 1933 and 1935 and the diverse versions of the model IS-LM, it shows that the problems arise from the way Keynes had approached this problem. To argue about the monetary value of the aggregates leads to dichotomize the model and does not allow to analyze correctly the interdependence between the labor market on one hand and the goods markets and the money market on the other hand. The model so formulated does not allow to treat in a rigorous way the effects of a variation of the monetary wage. It lets think, wrongly, that there is inevitably full employment equilibrium.

Mots clefs : Keynes, IS-LM, unité de mesure.

Keywords: Keynes, IS-LM, unit of measure.

JEL Classification: B22, E12

\footnotetext{
1 THEMA, Université de Cergy-Pontoise, F-95000 Cergy-Pontoise. E-mail: beraud@u-cergy.fr. Ce texte a été présenté au XIV Colloque Charles Gide, Histoire de la macroéconomie : des années de haute théorie (19261939) à nos jours. Université de Nice, 7-9 juin 2012. Je remercie les rapporteurs pour leurs remarques et suggestions.
} 


\section{Le problème}

Comme le note Michel de Vroey (2000, p. 293), l'étudiant qui, pour la première fois, lit l'article de John Hicks (1937) "Mr. Keynes and the "classics" » est surpris quand il comprend que le modèle SS-LM qui y est développé diffère sur bien des points du modèle IS-LM qu'il a étudié dans les manuels de macroéconomie : le chômage y apparaît aussi bien dans la version classique que dans la version keynésienne du modèle. Certes Hicks élabora l'appareil conceptuel qu'utilisèrent ses successeurs. Cependant ce n'est pas sa version que l'on retrouve dans le modèle IS-LM mais celle que Franco Modigliani (1944 a et b) élabora dans sa thèse et présenta dans un article intitulé « Liquidity preference and the theory of interest and money ». Modigliani avança, en particulier, l'idée que la différence cruciale entre les modèles keynésien et classique réside dans la flexibilité du taux de salaire monétaire et non, comme le soutenait Hicks, dans la théorie de la préférence pour la liquidité. Cependant certaines difficultés liées au choix de l'unité dans laquelle les grandeurs sont exprimées, déjà présentes dans l'article de Hicks, deviennent plus gênantes encore quand on cherche, comme Modigliani, à déterminer les effets d’une variation du taux de salaire monétaire.

Quand Franco Modigliani revint, en 1963, sur l'analyse qu'il faisait en 1944 du modèle keynésien, il nota qu'il convenait de corriger la formulation fautive qu'il avait, alors, retenue des propriétés d'homogénéité des fonctions de consommation, d'investissement et de demande de monnaie ${ }^{2}$.

"Je supposais ", écrivait-il, "que les trois fonctions de demande monétaire étaient homogènes de degré un dans le revenu monétaire seul. Cette formulation conduit à conclure à tort qu'une variation du revenu monétaire a le même effet sur la consommation monétaire qu'elle soit due à un changement du revenu réel à prix constants, ou à une variation des prix à revenu réel constant; et de même pour les deux autres variables. Cette erreur, à son tour, conduit à une propriété particulière du modèle, à savoir que les quatre premières équations du modèle forment un sous-système fermé en quatre variables $Y$ [le revenu monétaire], $S$ [la valeur de l'épargne], I [la valeur de l'investissement] et $r$ [le taux d'intérêt], comportant en particulier $M$ [la masse monétaire] comme paramètre. Cette dichotomie implique que les valeurs d'équilibre du taux d'intérêt et des variables de flux monétaires sont indépendantes des variables réelles du système, et en particulier de la forme de la fonction de production et du niveau auquel est fixé le salaire monétaire rigide $W$. Ceci cesse d'être vrai une fois que les fonctions de consommation et d'investissement sont

\footnotetext{
2 Nous ne suivrons pas Kouri (1986, p. 314) qui, rendant compte des contributions de Modigliani à la science économique, choisit d'ignorer cette erreur et lit l'article publié en 1944 comme si Modigliani l'avait écrit en 1963.
} 
formulées de façon adéquate ${ }^{3}$ [...] En particulier [...], pour une fonction de production donnée (y compris le stock initial de capital) et un $M$ donné, une hausse de $W$ tend normalement à provoquer une hausse de $r$, de même que de $P$, du revenu monétaire, de la valeur de l'investissement et de la consommation, et une baisse de l'emploi, du revenu réel et des autres variables de flux réels. » (Modigliani, 1963, p. 82)

En fait, il n'en est pas exactement ainsi. En 1944, Modigliani écrivait, dans le cas où le salaire monétaire est exogène, son modèle sous la forme suivante en notant $y$ le revenu réel, $p$ le niveau général des prix et $n$ l'emploi:

$$
\begin{aligned}
& M=L(r, Y) \\
& I=I(r, Y) \\
& S=S(r, Y) \\
& S=I \\
& Y \equiv p y \\
& y=f(n) \\
& W=p f^{\prime}(n)
\end{aligned}
$$

On peut difficilement interpréter ce modèle, comme Modigliani le suggérait en 1963, en supposant que les fonctions de demande " monétaires » sont homogènes de degré 1 dans le revenu monétaire. En effet, s'il en est ainsi, les taux d'épargne et d'investissement ne dépendent que du taux d'intérêt :

$$
\frac{I}{Y}=I(r, 1) \quad \frac{S}{Y}=S(r, 1)
$$

L'égalité des taux d'investissement et d'épargne détermine le taux d'intérêt qui ne dépend pas de la quantité de monnaie. Un tel modèle ne peut être présenté comme une interprétation de la pensée de Keynes si, du moins, comme Leijonhufvud (1994, p. 151), l'on pense que ce qui la caractérise c'est l'abandon de la théorie des fonds prêtables au profit de la théorie de la préférence pour la liquidité.

S'il est clair que Modigliani (1944 a, p. 33 ; 1944 b, p. 68) admettait que les fonctions de demande réelle sont homogènes de degré zéro dans les prix ${ }^{4}$, il ne prenait pas en compte cette

\footnotetext{
3 Modigliani précise, en note, que par adéquatement, il n'entend pas empiriquement correct mais simplement logiquement cohérent avec l'hypothèse qu'il cherche à formaliser.

${ }^{4}$ Modigliani ne déduit pas les propriétés d'homogénéité des fonctions de l'écriture des contraintes budgétaires si bien qu'il prétend qu'elles sont homogènes dans les seuls prix. Cette assertion est erronée puisqu'une variation des prix affecte la valeur des encaisses monétaires, des créances et des dettes. Il convient donc de dire que les fonctions d'offre et de demande réelles sont homogènes de degré zéro dans les prix et la valeur réelle de la position créditrice nette de l'agent. C'est cette idée que Patinkin développe dans sa thèse (1947:
} 
propriété dans l'écriture de son modèle. Sa formalisation mathématique ne correspond pas à son intention théorique. Ce qu'il supposait en 1944, dans sa thèse et dans l'article qui en est issu, c'est que les variations du revenu réel et des prix ont les mêmes effets sur la demande d'encaisses nominales, sur la valeur de l'investissement et sur l'épargne. C'est cette hypothèse qui pose problème car il convient, en général, de distinguer les effets d'une hausse du revenu réel et d'une hausse des prix. On peut d'ailleurs vérifier que les conclusions que Modigliani explicite - une variation de la valeur du salaire monétaire ou une modification de la fonction de production n'ont pas d'effet sur $Y, I, S$ et $r$ - sont indépendantes de toute hypothèse d'homogénéité et tiennent seulement à l'idée qu'une variation des prix a les mêmes effets qu'une variation du revenu réel.

La thèse de Modigliani, comme l'indique son titre, se veut une analyse de La Théorie Générale de l'emploi, de l'intérêt et de la monnaie sous les hypothèses de prix flexibles et de prix fixes. On doit donc se demander dans quelle mesure les difficultés qu'il a rencontrées et qu'il n'a pas pu surmonter, alors, trouvent leur origine dans l'ouvrage de Keynes qui discute la question de l'unité de mesure et conclut finalement que, pour élaborer une théorie de l'emploi, il convient de ne faire usage que de deux types de mesure des quantités, à savoir les valeurs monétaires et les quantités de travail (Keynes, 1936, p. 41). C'est à cette question que sera consacrée la première partie de cet article.

Modigliani (1944 a, p. 3) note que le modèle sur lequel il s'appuie s'inspire notamment des articles qu'Oskar Lange (1938) et Hicks (1937) avaient consacrés à ce sujet. Quand il s'agit de choisir l'étalon dans lequel les grandeurs sont mesurées, Lange et Hicks diffèrent. Keynes avait proposé deux solutions. Lange adopte la seconde : il mesure toutes les variables en quantités de travail commandé. Hicks suit la première : il s'en tient aux grandeurs monétaires. Modigliani (1944) et Lawrence Klein (1947) soutiennent l'équivalence des deux solutions. La thèse de Modigliani fut critiquée par Don Patinkin (1948 a, p. 153) mais son point d'attaque - la relation entre les propriétés d'homogénéité et la loi de Say - était telle qu'il n'était nullement évident de faire une relation entre elle et la formalisation de la théorie keynésienne que proposait Modigliani. On notera, cependant, que, dans sa thèse, Patinkin (1947) proposait un modèle macroéconomique qui échappe aux critiques que l'on peut adresser à Hicks et à Modigliani. Quand il discute et critique l'analyse que Pigou (1943) faisait de l'existence de l'équilibre stationnaire, il propose (1948 b, p. 549) un modèle macroéconomique où la demande réelle de biens est une fonction du revenu réel. Cependant la portée de ces contributions échappa à de nombreux économistes si bien que la confusion persista longtemps. C'est seulement en

p. 48) quand il soutient que « le niveau absolu des prix intervient dans les fonctions de demande excédentaire à travers son effet sur la valeur réelle des actifs existants». 
1960 que Modigliani tira parti d'une réédition de son article de 1944 pour mettre en garde le lecteur contre l'erreur qui avait été la sienne.

Mais quelle est la nature de l'erreur? Quelles en sont les conséquences ? Le problème est double. Il est d'abord théorique : doit-on raisonner en valeur ? En quantités de travail commandé ? Doit-on, au contraire, raisonner sur des variables réelles ? Mais, il est aussi « sociologique » dans ce sens que les difficultés que suscitait la lecture des articles de Hicks et de Modigliani n'étant pas clairement perçues, les économistes furent conduits, notamment dans leurs exposés pédagogiques, à retenir des hypothèses qui, plutôt que de résoudre le problème, l'évitaient. Bien souvent, ils admirent que les prix étaient fixes si bien que les variations des grandeurs monétaires reflètent parfaitement les variations des grandeurs réelles. Mais, en raisonnant ainsi, ils s'interdisaient d'analyser les effets sur l'emploi d'une variation des salaires monétaires ou des prix.

\section{Keynes du Traité à la Théorie Générale}

Durant la période qui sépare la publication du Traité de la rédaction de la Théorie générale, la pensée de Keynes a considérablement évolué sur deux questions, ici, centrales : le choix de l'unité de mesure et la modélisation de l'équilibre économique. Sur le premier point, alors que, dans le Traité, il distinguait, de façon assez traditionnelle, dans l'évolution de la valeur du produit, les variations des prix de celles des quantités, il abandonna cette position dans la Théorie Générale pour raisonner en valeur ou en travail commandé. Sur le second point, il abandonne les équations fondamentales du Traité pour proposer un modèle de détermination du niveau de l'emploi.

\subsection{Le choix de l'unité de mesure}

Keynes, dans le Traité, s'appuie sur la notion de pouvoir d'achat de la monnaie : " Puisque le pouvoir d'achat de la monnaie dans un contexte donné dépend de la quantité de biens et de services que peut acheter une unité de monnaie, il s'en suit qu'il peut être mesuré par le prix d'une marchandise composite, constituée de divers biens et services dans des proportions qui tiennent à leur importance en tant qu'objets de dépense. " (1930, t. 1, p. 47). L'indice du prix de cette marchandise composite est l'étalon dans lequel le pouvoir d'achat de la monnaie est mesuré. II cite (Ibid., p. 49), à cet égard, les principales analyses que Jevons, Edgeworth... ont faites de la construction des indices de prix ${ }^{5}$. Il est ainsi conduit, logiquement, à introduire dans son analyse la

\footnotetext{
5 On notera qu'il ne mentionne pas les articles que Divisia consacra à cette question. Sans doute l'intérêt que portait Keynes aux indices de prix était-il alors moindre qu'à l'époque, 1909, où il rédigea, sous la direction d’Alfred Marshall, son essai sur les nombres indices qui fut récompensé par le prix Adam Smith. S'il ignore
} 
notion de production globale. La quantité de produit, à une date $t$, est sa valeur estimée à son coût de production durant l'année de base (Ibid., t. 1, p. 121-2). Le problème des équations fondamentales est, alors, de distinguer dans les variations de la valeur du produit, l'évolution des prix de celle des quantités.

Dans les cours qu'il donna en 1932, Keynes continuait d'utiliser ces notions d'indice de prix et de volume de la consommation ou de l'investissement. Par exemple, dans le cours qu'il donna le 21 novembre 1932, il introduisait le prix des biens de consommation et celui des actifs (Rymes, 1989, pp. 77-9). Cependant, déjà, il hésitait : une semaine plus tard, il faisait référence à Adam Smith et à son idée que le travail est, par excellence, l'étalon de valeur mais il n'allait pas plus loin que ce rappel. L'année suivante, il franchit un nouveau pas (Ibid., p. 103). Il souligne que l'économiste doit définir précisément les termes qu'il emploie. Or, il a souvent affaire à un groupe complexe d'objets et il désire les réduire à des unités homogènes. Par exemple, il veut homogénéiser les divers biens qui composent le produit. II considère alors deux possibilités : raisonner en monnaie ou en termes d'emploi. Certes, le travail n'est pas homogène mais on pourra prendre pour unité le jour d'un travail ordinaire et considérer un travail spécialisé comme comptant pour deux unités, s'il vaut deux fois plus. Ainsi la question des indices n'est pas évacuée : elle apparaît à nouveau quand on veut homogénéiser le travail. Le plus souvent, Keynes raisonne alors en valeur ; mais quand il présente à ses étudiants le multiplicateur, il raisonne en quantités de travail. "Supposons que le revenu d'un homme commande le produit et l'emploi de $n$ [travailleurs] et supposons qu'il consomme un volume de produit et d'emploi $n_{1}$. Alors la relation $n_{1} / n$ est sa propension [moyenne] à consommer. Si son revenu s'accroît pour commander un produit et un emploi $n+\Delta n$ et que sa consommation s'accroît de $\Delta n_{1}$, alors $\Delta n_{1} / \Delta n$ est sa propension marginale à consommer [...] Le multiplicateur donne la relation entre la variation de l'emploi total à l'emploi résultant d'une augmentation de I'investissement. » (Ibid., p. 117).

Cette thèse est reprise, en deux temps, dans La Théorie Générale. Keynes reproche, d'abord, à Marshall et à Pigou de définir le dividende national comme une mesure du volume de la production courante ou comme un revenu réel et non comme la valeur du produit ou comme le revenu monétaire. Une telle définition n'est pas, selon lui, acceptable car « le produit de biens et services de la communauté est un ensemble complexe que l'on ne peut mesurer, à strictement parler, que dans certains cas spéciaux, comme par exemple quand tous les éléments d'un produit sont inclus dans les mêmes proportions dans un autre produit. » (Keynes, 1936, p. 38). Dès lors, Keynes propose, pour

Divisia, ce n'est pas parce qu'il est français mais parce qu'il n'a pas pris connaissance de la littérature la plus récente dans ce domaine. 
élaborer une théorie de l'emploi, de n'utiliser que deux types de quantité : les valeurs monétaires et l'emploi. L'offre et la demande globales seront ainsi définies comme des relations entre les valeurs de l'offre et de la demande d'un côté et l'emploi de l'autre. Si l'on note $Y_{s}$ les recettes (nettes du coût d'usage) attendues qui suscitent un emploi $n$, la fonction d'offre globale s'écrira $Y_{s}=\phi(n)$. De la même façon, si $Y_{d}$ sont les recettes que les entrepreneurs s'attendent à tirer de l'emploi de $n$ hommes, la fonction de demande globale s'écrira $Y_{d}=\psi(n)$. Souvent, cependant, Keynes (Ibid., p. 280) préfère raisonner sur des grandeurs définies en termes d'unité de salaire, soit si on note $W$ le taux de salaire monétaire, $\tilde{Y}_{s}=\frac{Y_{s}}{W}$ et $\tilde{Y}_{d}=\frac{Y_{d}}{W}$.

Pour interpréter l'évolution de la pensée de Keynes du Traité à la Théorie Générale, il faut distinguer trois questions qui sont certes liées mais différentes: l'agrégation, le choix de l'unité de mesure et l'interprétation des équations. Keynes est de plus en plus sensible aux difficultés qu'implique l'agrégation de grandeurs hétérogènes; ceci le conduit à mettre en doute le sens qu'il convient de donner à des grandeurs comme le produit global. Dans le Traité, Keynes utilisait comme étalon de valeur, un ensemble donné de biens. Plus tard, il écarte cette mesure. Deux étalons possibles sont alors proposés : la monnaie et le travail. Il dit à ses étudiants que les équations sur lesquelles il s'appuie dans ses cours sont « symboliques plutôt qu'algébriques » (21 novembre 1932, in Rymes, 1989, p. 77), qu'elles sont simplement des illustrations (4 décembre 1933, Ibid., p. 126). Ce ne sont pas, à proprement parler, des équations mathématiques mais plutôt une façon de noter brièvement, en sténo pourrait-on dire, les relations entre des ensembles complexes de variables. Pour passer de ces notations à des équations algébriques, il faudrait introduire des hypothèses qui semblent, à Keynes, trop restrictives.

C'est, peut-être, le débat suscité par la publication du Traité de la monnaie qui explique pourquoi Keynes porta, dans les années trente, une attention nouvelle aux difficultés qu'implique l'agrégation. Dans le Traité (1930, t. 1, 114), il expliquait que, par valeur de l'investissement, il entendait non l'augmentation de la valeur du capital total mais la valeur de l'accroissement du capital. Hayek (1931, p. 281) trouva cette formulation ambiguë car l'on ne peut parler d'une augmentation de la quantité de capital que si, aux deux dates considérées, le capital se compose des mêmes biens pris dans les mêmes proportions. Keynes (1931, p. 397), dans la réponse qu'il fit alors, ne considérait pas qu'il y avait là une difficulté nouvelle. Le problème, écrivait-il, est le même que celui qui se pose quand on calcule un indice de prix et un taux de salaire réel alors même que la composition du panier de biens consommé par les salariés a changé. Il faut croire qu'à la réflexion, la difficulté lui apparut plus fondamentale : on ne peut pas mesurer, à strictement parler, l'évolution de 
quantité de capital si sa composition a, au cours du temps, évolué. Mais un autre facteur a joué : du Traité à la Théorie générale l'objet de l'étude a changé. Dans le Traité de la monnaie, il est question de la dynamique des prix. Keynes ne pouvait, pour en parler, se passer de la notion de niveau général des prix. II était ainsi conduit à distinguer dans la valeur du produit, sa quantité et son prix. Dans la Théorie générale, il étudie la détermination du niveau de l'emploi et le recours à la notion de la quantité de produit global n'est plus, selon lui, nécessaire. Il décida donc de s’en passer.

\subsection{Le modèle de Keynes}

Keynes, dans le cours qu'il donna le 4 décembre 1933, expliquait à ses étudiants un modèle très voisin de celui qui, selon Hicks, décrit la pensée keynésienne (Dimand, 2007, pp. 85-6). II s'en écarte toutefois sur un point : dans chaque fonction, Keynes inclut comme argument "the state of news" pour montrer que la demande de monnaie, les dépenses de consommation et d'investissement dépendent de ce que les agents savent, ou croient savoir, de la situation économique et de son évolution. II introduit ainsi dans son modèle les anticipations et l'incertitude.

Il écrit, d'abord, le modèle en valeur. Son analyse de la préférence pour la liquidité s'exprime comme une relation entre la quantité de monnaie, $M$, le taux d'intérêt, $r$, et les informations disponibles, $\xi$ :

$$
M=L(r, \xi)
$$

Le revenu monétaire, $Y$, est la somme des dépenses de consommation, $C$, et d'investissement $I$ :

$$
Y=C+I
$$

Les dépenses de consommation sont une fonction du revenu monétaire et des informations disponibles :

$$
C=C(Y, \xi)
$$

Les dépenses d'investissement sont fonction du taux d'intérêt et de l'état des informations disponibles :

$$
I=I(r, \xi)
$$


De cette formulation en valeur, il passe, sans explication, à une formulation en quantités de travail. Soit $n$ le nombre total de personnes employées, $n_{c}$ le nombre de celles qui produisent des biens de consommation et $n_{i}$ de celles qui produisent des biens durables, on a

$$
\begin{aligned}
& n_{c}=\gamma(n) \\
& n_{i}=\imath(r) \\
& n=n_{c}+n_{i}
\end{aligned}
$$

Un de ses étudiants, Robert Bryce, note que le taux d'intérêt dépend de la quantité de monnaie mesurée en unités de salaire :

$$
\iota(r)=\lambda\left(\frac{M}{W}\right)
$$

Ce qui laisse Rymes (1989, p. 126) dubitatif. Le lecteur reste perplexe, car Keynes juxtapose deux modèles différents. Dans le premier, la quantité de monnaie détermine le revenu monétaire et le taux d'intérêt. Dans le second, la masse monétaire mesurée en unités de salaire détermine le taux d'intérêt et le niveau de l'emploi. On est tenté de l'interpréter en disant qu'une augmentation de la quantité de monnaie et une baisse du salaire monétaire accroissent de la même façon le niveau de l'emploi comme l'expliquera, trente ans plus tard, Modigliani (Modigliani, 1963, p. 89).

On retrouve une formulation comparable dans le projet du chapitre 9 de la Théorie Générale qui étudie les relations entre l'emploi, la consommation et l'investissement (Keynes, décembre 1933 in Keynes, 1971-1989, t. 13, p. 439 et suivantes). La consommation et l'investissement sont, alors, mesurés en unités de salaire et sont présentés comme des fonctions de l'emploi, du taux d'intérêt et de l'état des anticipations de long terme. Cette idée est reprise dans une nouvelle version datée 1934. En notant $\tilde{C}$ et $\tilde{l}$ la consommation et l'investissement mesurés en unités de salaire, $F_{c}$ et $F_{i}$ les fonctions d'emploi pour les biens de consommation et d'investissement, Keynes, dans une situation où une partie des travailleurs sont au chômage, analyse l'emploi comme une fonction de l'état des anticipations et du taux d'intérêt :

$$
\begin{aligned}
& \tilde{C}=\tilde{C}(n, r, \xi) \\
& \tilde{l}=\tilde{l}(n, r, \xi) \\
& n_{c}=F_{c}(\tilde{C}) \\
& n_{i}=F_{i}(\tilde{l}) \\
& n=n_{i}+n_{c}
\end{aligned}
$$


Il est déterminé par la demande de biens.

La reformulation de la théorie de la préférence pour la liquidité est plus tardive. Dans le cours qu'il donna le 25 novembre 1935, Keynes (in Rymes, 1989, p. 178) distingue quatre motifs de détention de la monnaie : le revenu, les affaires, la précaution et la spéculation. Il scinde alors la fonction de liquidité en opposant $L_{1}$ qui renvoie au trois premiers motifs et qui serait fonction du revenu monétaire et $L_{2}$ qui dépendrait principalement du taux d'intérêt :

$$
M=L_{1}(Y)+L_{2}(r)
$$

Keynes reprend ces idées, de façon peut-être moins explicite, dans la Théorie Générale en opposant la fonction d'offre globale - ou la fonction d'emploi - à la fonction de demande globale. Quand il explique le principe de la demande effective (1936, p. 25), il raisonne en valeur. Le prix d'offre du produit global apparaît comme une fonction de l'emploi, $Y_{s}=Y_{s}(n)$. La fonction de demande globale décrit les recettes que les entrepreneurs espèrent tirer de l'emploi de $n$ travailleurs : $Y_{d}=Y_{d}(n)$. Cependant, quand il étudie la fonction de consommation, il préfère raisonner en termes de travail commandé. "Puisque nous sommes ici concernés par la détermination de la somme qui sera dépensée pour la consommation quand l'emploi est à un niveau donné, nous devrions, à proprement parler, considérer la fonction qui relie la première quantité $(C)$ à la seconde (n). Il est plus commode, cependant, de travailler en termes d'une fonction légèrement différente qui relie la consommation en termes d'unités de salaires $(\tilde{C})$ au revenu en termes d'unité de salaires $(\tilde{Y})$ correspondant au niveau de l'emploi $n$. » (Keynes, 1936, p. 90). Keynes admet, certes, qu'à un volume donné de l'emploi puisse correspondre, selon la nature de celui-ci, divers niveaux de $\tilde{Y}$ et qu'il faille, dans certains cas, prendre en compte ces différences. Cependant, il soutient que considérer $\tilde{Y}$ comme une fonction du seul niveau de l'emploi est généralement approprié. Ce que Keynes ne nous dit pas, c'est pourquoi il est plus « commode » de raisonner sur la relation entre $\tilde{C}$ et $\tilde{Y}$ plutôt que sur la relation entre $C$ et $n$.

\section{Les keynésiens}

Certains économistes anglais qui avaient été très proches de Keynes — Joan Robinson (1971, p. 143), par exemple - ont présenté le modèle IS-LM comme le point de départ de la critique que les économistes orthodoxes firent de l'œuvre de Keynes. À l'inverse, Paul Samuelson (1946, p. 188) a soutenu que " jusqu'à l'apparition des modèles mathématiques de Meade, Lange, Hicks et Harrod, on avait des raisons de penser que Keynes lui-même ne comprenait pas sa propre analyse ". Comme 
nous l'explique Dimand (2007), les uns et les autres ont tort. Clairement, avant que Hicks écrive son article, Keynes avait conçu un modèle de type IS-LM et il l'expliquait à ses étudiants. Certains d'entre eux - Brian Reddaway (1936) et David Champernowne (1936) - s'appuyèrent sur ses leçons pour rendre compte de la Théorie Générale 6 . L'un et l'autre assistèrent, en particulier, à la leçon du 4 décembre 1933 durant laquelle, comme on l'a expliqué plus haut, Keynes exposa une version algébrique de son modèle. Ils y prirent des notes dont ils ne manquèrent pas de se servir. Leurs articles sont particulièrement importants puisqu'ils furent publiés avant la réunion de l'Econometric Society où Hicks, Meade et Harrod présentèrent les premières versions de leurs modèles et l'on peut penser que Hicks, qui était rédacteur de la Review of Economic Studies où l'article de Champernowne fut publié, en avait eu connaissance (Young, 1987, pp. 82-3). Lange (1938, p. 12) fait explicitement référence à l'article de Reddaway en lui attribuant le mérite d'avoir, avant lui, conçu un système d'équations similaire à celui sur lequel il s'appuie. Bref, le modèle IS-LM trouve son origine dans l'enseignement de Keynes. Cela n'implique pas que l'interprétation qu'il propose de la Théorie Générale ne puisse pas être critiquée. On a vu que Keynes, lui-même, mettait en garde ses étudiants : les relations qu'il écrivait étaient plutôt des illustrations que des équations algébriques. Pire, il hésitait, passant d'une écriture à l'autre sans démontrer l'équivalence des diverses formulations sur lesquelles son enseignement s'appuyait. Faut-il soutenir que la valeur de la consommation dépend du niveau de l'emploi ou qu'elle est fonction du revenu monétaire ? Doit-on plutôt écrire que la quantité de travail que commandent les dépenses de consommation est fonction de la quantité de travail que le revenu commande ? Sans surprise, les lecteurs de Keynes optèrent, sans vraiment justifier leur choix, pour telle ou telle écriture : certains raisonnèrent en valeur, d'autres en travail commandé, d'autres en quantités physiques. Champernowne introduisit le niveau de l'emploi comme argument des fonctions d'épargne, d'investissement et de demande de monnaie. Reddaway ne stipula pas explicitement I'unité de mesure qu'il utilisait. II semble, cependant, raisonner en valeur. À la réflexion, Modigliani (1944 a, p. 32, 1944, p. 67) et Klein (1947, p. 202) prétendirent que cela revenait au même tandis que Patinkin (1947) réintroduisait dans son raisonnement la notion de produit réel, passant ainsi outre à la critique que Keynes faisait, parfois, de cet agrégat.

\footnotetext{
6 Citons, sur ce point, Tortajada (2009, p. 51) « Champernowne rédigea et proposa son article à la publication avant que ne paraisse le Théorie Générale... L'article visait à mettre en évidence le noyau logique des Conférences de Keynes en 1934 et 1935 à la "Saint-Michel" [...] où celui-ci exposait le résultat de ses recherches. » Young $(1987$, p. 83) rapporte à cet égard le témoignage de Champernowne qui lui disait que son article « reposait sur les conférences de Keynes et son tutorat. »
} 


\subsection{Raisonner en valeur}

On peut réécrire le modèle qui, chez Hicks (1937, p. 156), détermine le revenu monétaire et le taux d'intérêt sous la forme suivante :

$$
\begin{aligned}
& M=L(Y, r) \\
& I=I(Y, r) \\
& S=S(Y, r) \\
& I=S
\end{aligned}
$$

Ces quatre équations permettent de calculer le revenu monétaire, $Y$, le taux d'intérêt, $r$, la valeur de l'investissement, $I$, et la valeur de l'épargne $S$ en fonction de la quantité de monnaie. Hicks explique longuement pourquoi il introduit le revenu comme argument des fonctions d'investissement et de demande de monnaie. Il évoque l'élégance mathématique qui impliquerait un traitement symétrique des trois fonctions ; mais le problème n'est pas l'élégance de l'analyse mais sa cohérence logique. On ne peut guère soutenir que des agrégats monétaires, comme la valeur de l'investissement ou la demande d'encaisses nominales, dépendent du seul taux d'intérêt. II y aurait, dans ce cas, une difficulté conceptuelle puisqu'une variation des prix resterait sans effet sur la demande de monnaie et les dépenses d'investissement. Mais, c'est principalement sur deux points qu'il faut attirer l'attention : la forme des fonctions de demande de biens et le comportement du modèle dans une situation de plein emploi.

L'élasticité des fonctions de demande réelle de biens ${ }^{7}$ par rapport aux prix est égale à - 1 et ces fonctions peuvent être représentées par des branches d'hyperbole équilatère. Ainsi, il existe, toujours, un équilibre : quand le prix tend vers zéro, la demande tend vers l'infini ; quand le prix tend vers l'infini la demande tend vers zéro. En particulier, si le salaire monétaire est flexible 8 , il existe toujours un équilibre de plein emploi. Le plus curieux, c'est qu'il en est ainsi même dans le cas particulier keynésien, où, dans la spécification de Hicks, la première équation du système (1) se réécrit $M=L(r)$. La masse monétaire détermine le taux d'intérêt qui ne peut descendre en-dessous d'un niveau $r_{\min }$. Supposons que l'économie est dans une trappe à liquidité et que le taux d'intérêt soit $r_{\min }$. Les trois dernières équations du système (1) déterminent les valeurs de l'investissement $I\left(r_{\min }\right)$, de la consommation $C\left(r_{\min }\right)$ et du revenu $Y\left(r_{\min }\right)$. Supposons que le taux de salaire monétaire

\footnotetext{
7 On peut déterminer simplement les dépenses de consommation, $C$, comme égales à la différence entre le revenu monétaire $Y$ et l'épargne $S$. On a, donc, $C=C(Y, r)$.

${ }^{8}$ Certes, Hicks raisonne le plus souvent avec un salaire monétaire donné et l'on peut soutenir, comme le fait Ingo Barens (1999, p. 88), que sous cette hypothèse, le revenu monétaire peut servir d'estimation du revenu réel et de l'emploi.
} 
soit flexible. Il existe toujours un niveau du taux de salaire monétaire, $W$, des prix de consommation, $p_{c}$, et des prix d'investissement, $p_{i}$, qui assurent un équilibre de plein emploi. La trappe à liquidité ne fait rien à l'affaire.

Explicitons ce résultat. On peut décrire l'équilibre sur le marché des biens et le marché du travail par les équations suivantes en admettant, contrairement à Hicks, que le taux de salaire monétaire, $W$, est une variable endogène et que l'offre de travail, $n$, est donnée :

$$
\begin{aligned}
& I=p_{i} f_{i}\left(n_{i}\right) \\
& W=p_{i} f_{i}{ }^{\prime}\left(n_{i}\right) \\
& C=p_{c} f_{c}\left(n_{c}\right) \\
& W=p_{c} f_{c}{ }^{\prime}\left(n_{c}\right) \\
& n=n_{i}+n_{c} .
\end{aligned}
$$

$n_{i}$ et $n_{c}$ sont l'emploi respectivement dans le secteur des biens d'investissement et dans celui des biens de consommation. $f_{i}$ et $f_{c}$ sont les fonctions de production supposées continues et croissantes. Leurs dérivées premières sont continues décroissantes et tendent vers zéro quand l'emploi tend vers l'infini et vers l'infini quand l'emploi tend vers zéro. Les deux premières équations permettent de montrer que la demande de travail dans le secteur des biens d'investissement est une fonction continue décroissante du taux de salaire monétaire $n_{i}=n_{i}^{d}(I / W)$ et que $n_{i}$ tend vers l'infini quand $W$ tend vers zéro et que $n_{i}$ tend vers zéro quand $W$ tend vers l'infini. On obtient le même résultat dans le secteur des biens de consommation. La demande totale de travail est, pour un niveau donnée des dépenses d'investissement et de consommation, une fonction monotone décroissante du salaire monétaire qui tend vers l'infini pour $W$ tendant vers zéro et qui tend vers zéro quand $W$ tend vers l'infini. L'équilibre sur le marché du travail défini le taux de salaire monétaire d'équilibre et le niveau de l'emploi dans les deux secteurs. 


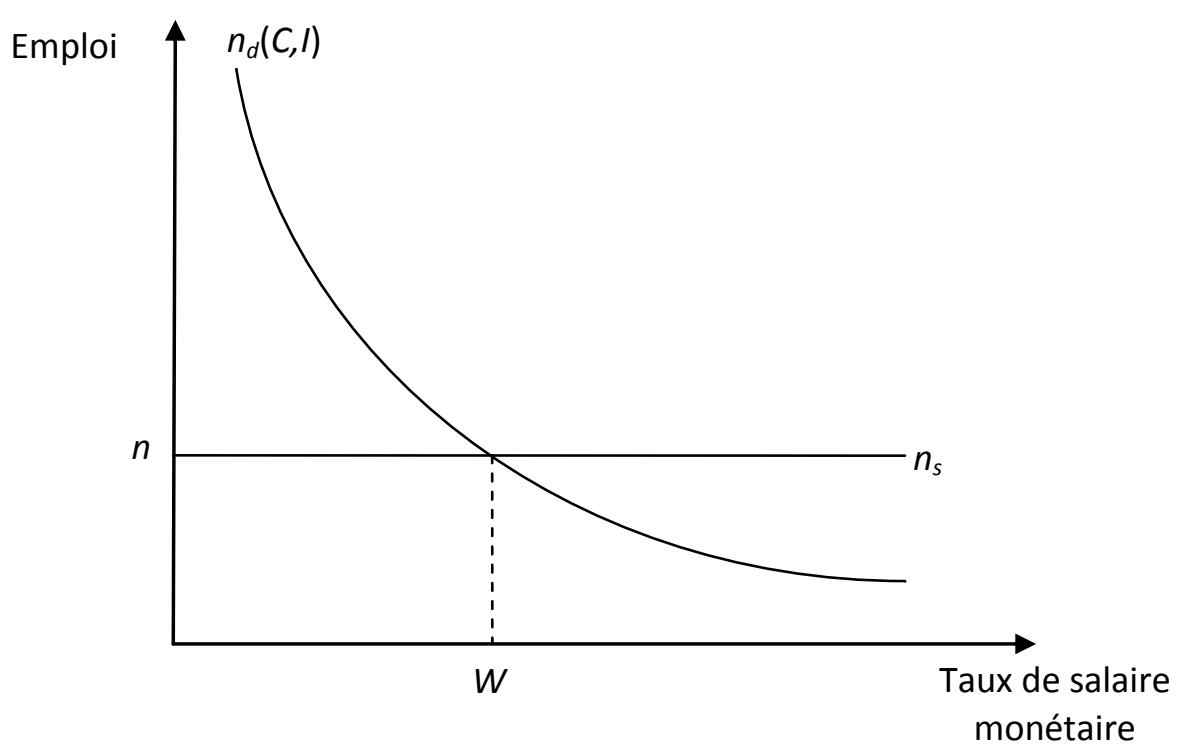

Figure 1 : L'équilibre sur le marché du travail

Soit $f_{i}\left(n_{i}\right)$ et $f_{c}\left(n_{c}\right)$ l'offre de biens d'investissement et de consommation. La demande de biens de consommation est $c_{d}=C\left(r_{\text {min }}\right) / p_{c}$ où $p_{c}$ est le prix des biens de consommation. Elle est représentée par une branche d'hyperbole équilatère. La demande de biens de consommation tend vers l'infini quand $p_{c}$ tend vers 0 et vers 0 quand $p_{c}$ tend vers l'infini. II existe donc toujours un prix positif $p_{c}$ tel que l'offre de biens de consommation soit égale à la demande de ces biens.

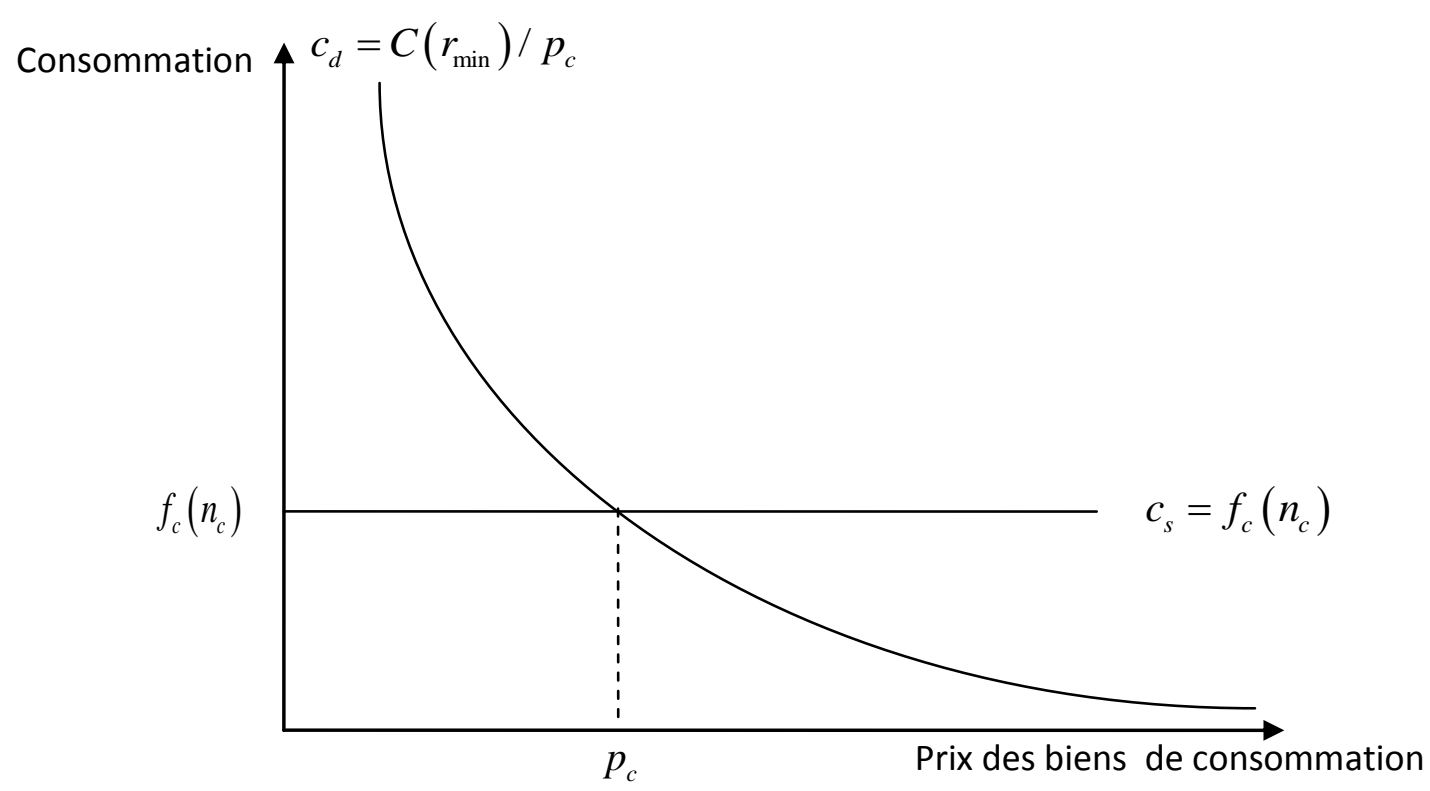

Figure 2 : L'équilibre sur le marché des biens de consommation dans le modèle de Hicks

Le même résultat peut être obtenu pour le secteur des biens d'investissement, si bien que I'on peut assurer que, sous des hypothèses relatives aux propriétés des fonctions de production et à 
la concurrence qui sont celles de Hicks, il existe un équilibre de plein emploi même dans le cas d'une trappe à liquidité.

Pour analyser la cohérence logique de la théorie quantitative de la monnaie et les effets d'une variation des salaires monétaires, Modigliani (1944 a, p. $32 ; 1944$ b, p. 68) réécrit son modèle de façon à raisonner non sur des valeurs monétaires mais des quantités de travail commandé. ॥ soutient, alors, qu'il y a un cas où la théorie keynésienne de la préférence pour la liquidité suffit à expliquer l'existence d'un équilibre de sous-emploi sans qu'il soit nécessaire de faire référence à la rigidité des salaires monétaires. Puisque, nous dit-il, les titres sont une forme de détention des actifs inférieure à la monnaie, il doit exister un niveau positif du taux d'intérêt, $r_{\min }$, pour lequel la demande de monnaie devient infiniment élastique. Les équations

$$
\begin{aligned}
& \tilde{I}=\tilde{I}\left(\tilde{Y}, r_{\text {min }}\right) \\
& \tilde{S}=\tilde{S}\left(\tilde{Y}, r_{\text {min }}\right)
\end{aligned}
$$

suffisent, alors, pour déterminer le revenu mesuré en unités de salaire. Mais cette valeur n'est généralement pas compatible avec le taux de salaire réel et le niveau de l'emploi qu'implique l'équilibre du marché du travail. "Plus de travailleurs souhaiteraient travailler au taux de salaire réel courant qu'il n'y en a d'employés ; mais les efforts pour réduire le salaire réel et augmenter l'emploi sont voués à l'échec. Car toute baisse des salaires et des prix accroît l'offre de monnaie pour la spéculation mais ne peut pas diminuer le taux d'intérêt [...] puisque la demande de monnaie pour la spéculation est infiniment élastique. " (Modigliani, 1944 a, p. 39, 1944 b, p. 74). Pour mettre en évidence ce résultat, Modigliani abandonne le raisonnement en termes de grandeurs monétaires, il mesure tous les agrégats en termes de travail commandé, il évite ainsi l'erreur de Hicks.

Une seconde difficulté apparaît quand Hicks étudie les rapports entre les analyses de Keynes et de Wicksell. Après avoir introduit le revenu monétaire dans la fonction d'investissement, il trace les courbes représentatives de cette fonction et de la fonction d'épargne dans un graphique où le taux d'intérêt figure en ordonnée et l'investissement et l'épargne en abscisse. Une augmentation du revenu monétaire accroît, à la fois, l'épargne et l'investissement pour un taux d'intérêt donné. L'idée qu'avance Hicks est, qu'en plein emploi, il est possible que l'épargne et l'investissement augmentent du même montant quand le revenu monétaire s'accroît. Bien que, sur ce point, Hicks ne soit pas explicite ${ }^{9}$, on imagine qu'il ne peut en être ainsi que dans le cas où la hausse du revenu monétaire est purement nominale et où l'augmentation des prix accroît dans les mêmes proportions l'épargne

\footnotetext{
${ }^{9}$ Modigliani (1944 b, p. 59) est, sur ce point, plus clair.
} 
et l'investissement parce que ses fonctions sont homogènes de degré 1 dans les prix. La courbe IS est horizontale quand l'économie a atteint le plein emploi. On a alors, écrit Hicks (1937, p. 158) une construction parfaitement wicksellienne. Le taux d'intérêt est déterminé par des causes réelles et son niveau peut être qualifié de naturel. Si le taux monétaire est fixé en dessous de ce taux, une inflation cumulative se développe; s'il est fixé au-dessus, une déflation cumulative se produira. Cependant ce raisonnement implique que les valeurs de l'épargne et de l'investissement sont des fonctions homogènes de degré 1 dans les prix. S'il en est ainsi, il faut prendre en compte cette propriété dans leur écriture et dans la résolution du modèle.

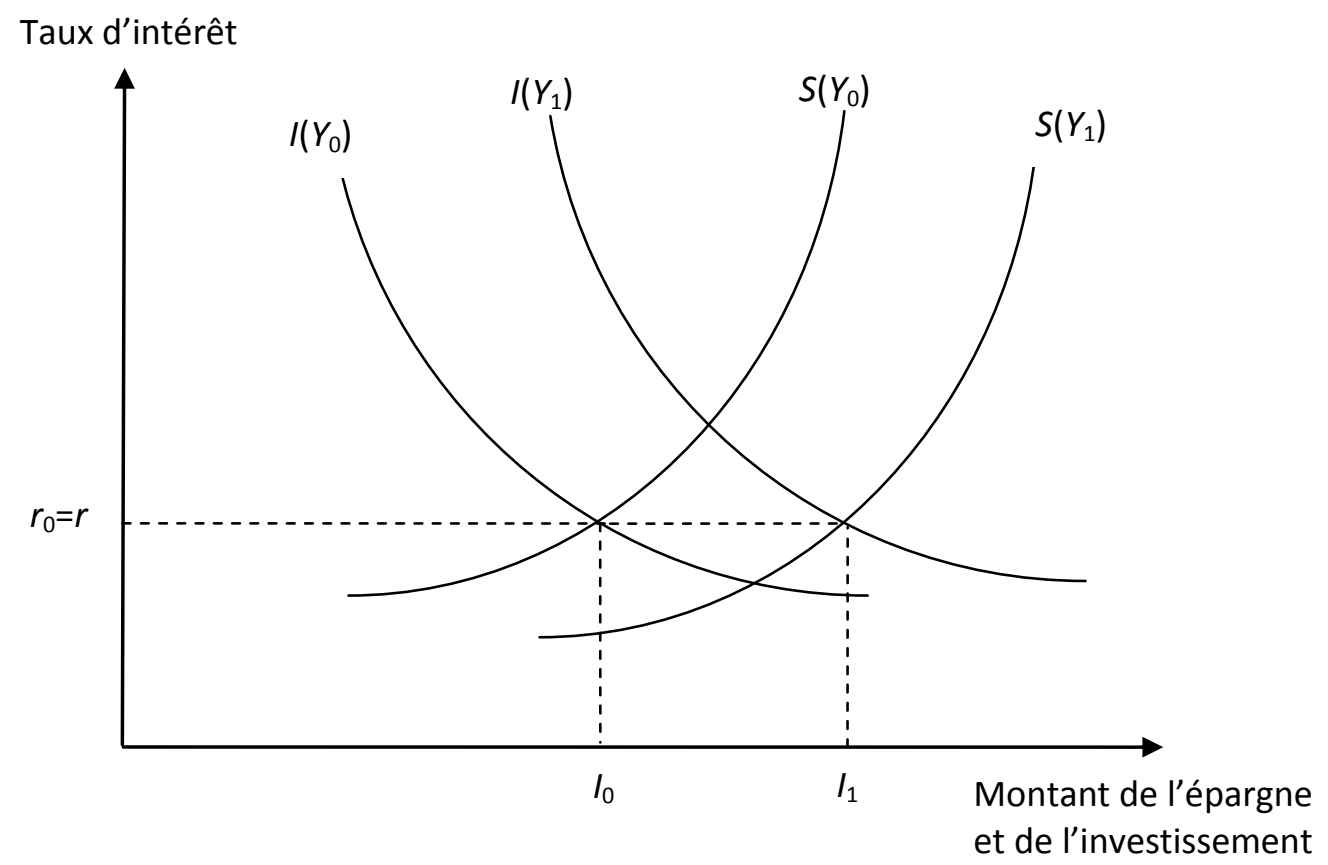

Figure 3 : L'effet d'une hausse du revenu monétaire sur l'investissement (Hicks, 1937, p. 157)

\subsection{Le travail commandé}

Lange mesure en travail commandé la valeur de tous les agrégats qu'il introduit dans son système : le revenu, la consommation, l'investissement mais aussi la masse monétaire. En surlignant par un tilde les grandeurs exprimées en unités de salaire, on obtient :

$$
\begin{aligned}
& \frac{M}{W}=\tilde{L}(r, \tilde{Y}) \\
& \tilde{C}=\tilde{C}(r, \tilde{Y}) \\
& \tilde{I}=\tilde{I}(r, \tilde{C}) \\
& \tilde{Y}=\tilde{C}+\tilde{I}
\end{aligned}
$$


Il soutient que le choix de l'étalon est arbitraire : il a choisi le travail mais n'importe quel autre numéraire aurait aussi bien fait l'affaire. Il fait référence aux articles de Reddaway et de Hicks en soulignant la parenté des approches mais sans vouloir expliquer les différences d'écriture puisqu'il se borne à indiquer que "la forme choisie dans mon article semble, cependant, mieux adaptée à l'étude des problèmes qu'il traite » (Lange, 1938, p. 12).

Cependant, le commentaire que fait Lange de son équation d'équilibre du marché de la monnaie peut surprendre. Cette écriture suppose que « le rapport du prix de chaque marchandise ou service au prix de la marchandise ou du service qui a été choisi comme numéraire est donné. Ces rapports peuvent être considérés comme déterminés par un système walrassien ou parétien d'équations d'équilibre général. Ainsi les nombres indices ne sont pas impliqués dans cette procédure. » (Ibid., p. 13). Dans ce commentaire, trois propositions attirent l'attention. Le revenu qui apparaît dans le modèle de Lange est un revenu réel et on est tenté de le considérer comme un indice du volume du produit. C'est cette interprétation que Lange écarte en affirmant que les nombres indices ne sont pas impliqués dans la procédure. Faut-il y voir le souvenir des critiques adressées par Keynes à cette notion ? Peut-être, mais pas nécessairement. En effet, alors qu'habituellement on admet que les prix relatifs varient, Lange les suppose fixes. Dès lors, les problèmes traditionnels posés par l'agrégation de quantités hétérogènes sont éliminés. Supposons qu'il existe dans l'économie étudiée $k$ biens ou services que nous noterons $j$ et du travail que l'on peut bien considérer comme homogène si les salaires relatifs restent fixes. Le revenu en termes d'unités de salaire est défini par l'équation

$$
\tilde{Y}=\sum_{j=1}^{k} \frac{p_{j}}{W} y_{j}
$$

Tant que les prix relatifs, $p_{j} / W$, sont constants, les variations de $\tilde{Y}$ reflètent les variations des quantités de biens $j$ qui ont été produites (Rubin, 2007, p. 3). Nul recours à la théorie des nombres indices n'est nécessaire, le problème est que l'hypothèse de fixité des prix relatifs est restrictive. On notera qu'elle implique que le taux de salaire réel est fixe. Lange raisonne en unités de salaire ; mais comme il suppose que la quantité de travail que commande une marchandise est fixe, il aurait tout aussi bien pu raisonner en quantités physiques.

On remarquera, enfin, que Lange, quand il représente les variations de la consommation en fonction du revenu, trace une droite qui passe par l'origine. II ne discute pas et ne justifie pas ce graphe mais celui-ci laisse à penser qu'il admet qu'une variation du revenu mesuré en unités de salaire entraîne une variation proportionnelle de la consommation. Faut-il en conclure que la 
fonction de consommation est homogène de degré 1 dans le revenu réel dont les variations auraient sur les dépenses de consommation les mêmes effets qu'une variation des prix ? Raisonner en valeur ou en travail commandé reviendrait alors au même.

\subsection{Peut-on passer d'un raisonnement en valeur à un raisonnement en unités de} salaires?

C'est cette proposition que certains keynésiens, notamment Modigliani et Klein, cherchèrent à approfondir. Dans la Théorie Générale, Keynes passe, comme on l'a vu, d'un raisonnement en valeur à un raisonnement en unités de salaire. Sous quelles conditions peut-on passer de l'une à l'autre ? Hicks, comme on l'a vu, avait soutenu, avec circonspection ${ }^{10}$, l'idée que, dans une situation de plein emploi, une hausse du revenu nominal a les mêmes effets sur l'épargne et l'investissement et laisse inchangé le taux d'intérêt. Modigliani (1944 a, p. 32, 1944 b, p. 67) reformule cette idée en soutenant qu'une variation des prix affecte le revenu, l'investissement et l'épargne dans les mêmes proportions. Si les prix futurs anticipés varient comme les prix courants, les fonctions d'offre et de demande réelles de marchandises et de travail sont homogènes de degré zéro dans les prix. Klein (1949, p. 201) reprendra à son compte cette idée. « Ce système est homogène d'ordre zéro dans les prix dans toutes les équations sauf celle qui traite des encaisses monétaires. Si l'on change tous les prix et les salaires dans les mêmes proportions, l'on ne change aucune grandeur réelle dans des équations homogènes. » On peut, par exemple, écrire

$$
\left\{\begin{array} { l } 
{ M = L ( Y , r ) } \\
{ I = I ( Y , r ) } \\
{ S = S ( Y , r ) } \\
{ I = S }
\end{array} \quad \Rightarrow \quad \left\{\begin{array}{l}
M=L\left(W \frac{p y}{W}, r\right) \\
\frac{I}{W}=I\left(\frac{p y}{W}, r\right) \\
\frac{S}{W}=S\left(\frac{p y}{W}, r\right) \\
\frac{I}{W}=\frac{S}{W}
\end{array}\right.\right.
$$

En admettant que l'économie est en plein emploi, l'équilibre sur le marché du travail détermine le niveau de l'emploi et le taux de salaire réel ; indirectement, il permet de calculer le produit réel. Alors l'égalité de l'épargne et de l'investissement détermine le taux d'intérêt et

\footnotetext{
10 " II n'est pas invraisemblable », écrit Hicks (1937, p. 158), " qu'une hausse des salaires puisse entraîner le sentiment que les salaires augmenteront, à nouveau, ultérieurement. ". S'il en était ainsi, la hausse de l'investissement excéderait celle de l'épargne et la courbe IS serait, quand le plein emploi est atteint, croissante. Autrement dit, pour qu'une hausse des prix ait le même effet sur l'investissement et sur l'épargne, il faut que l'élasticité des anticipations soit égale à 1 , autrement dit que les prix futurs anticipés varient comme les prix courants laissant inchangé le taux d'inflation anticipé.
} 
l'équilibre sur le marché de la monnaie nous donne le taux de salaire monétaire. Les résultats classiques apparaissent clairement avec la dichotomie qui les caractérise. Les variables réelles l'emploi, le produit, la consommation, l'investissement et le taux d'intérêt - ne dépendent pas de la quantité de monnaie. Celle-ci n'intervient que pour déterminer le niveau des prix.

La discussion prit alors un cours curieux car Modigliani peina à rendre son analyse compatible avec les conclusions que Lange (1942) tirait de sa critique de la loi de Say. Lange soutenait d'une part que les fonctions d'offre et de demande pour les marchandises sont - quand la loi de Say s'applique - homogènes de degré zéro et d'autre part que cette loi est incompatible avec toute théorie monétaire. Modigliani chercha à convaincre ses lecteurs que les propriétés d'homogénéité des fonctions d'offre et de demande n'ont rien à voir avec la loi de Say et ne dépendent que de la rationalité des agents et de l'homogénéité de degré un des fonctions d'anticipation ${ }^{11}$. Que la question ait été posée sous cette forme explique pourquoi elle interférera avec la controverse que suscitera la mise en avant par Pigou (1943) de l'effet d'encaisse réelle. II apparaîtra alors que, pour que la variation des prix laisse inchangées les demandes nettes de biens, il faut que la valeur des actifs nets détenus par les agents varie comme les prix.

Le problème est en fait plus simple. Si, comme l'admet Modigliani, les fonctions de demande monétaire sont homogènes de degré 1 dans les prix, alors l'écriture qu'il adopte implique qu'elles sont homogènes de degré 1 dans le revenu monétaire; mais, il n'avance aucun argument pour expliquer qu'elles obéissent à cette propriété. Pire, s'il en est ainsi, on peut affirmer que le taux d'intérêt est déterminé sur le marché des biens et ne dépend ni de l'offre de monnaie ni de sa demande. Si on veut construire un modèle keynésien, c'est une hypothèse qu'il faut écarter.

Mais une autre question se pose. Pourquoi serait-il préférable de prendre comme numéraire le travail plutôt qu'un autre bien ? En 1944, Modigliani n'avance, sur ce point, aucun argument. En 1963, il soutient « que le principe keynésien d'utiliser le travail comme numéraire est très naturel puisque le salaire rigide constitue une unité stable de mesure. " (Modigliani, 1963, p. 89). C'est I'inverse qui est vrai. Diviser par une constante est inutile. Quand le taux de salaire monétaire est constant, on peut raisonner en valeur. Quand il varie, le prendre pour étalon a un sens mais ce choix n'est pas nécessairement approprié. II faut se demander si l'on ne doit pas passer outre les scrupules de Keynes et réintroduire dans le raisonnement la notion de revenu réel.

\footnotetext{
11 Ce qui est frappant dans son approche, c'est que jamais, en 1944, il ne s'appuie sur l'écriture de la contrainte budgétaire des agents pour analyser les propriétés d'homogénéité des fonctions d'offre et de demande.
} 


\subsection{La réintroduction de la notion de revenu réel}

Patinkin proposa dans sa thèse ${ }^{12}$ une solution à ces différentes énigmes, solution qui s'appuie clairement sur la tradition walrassienne. Son premier souci est de comprendre comment introduire le revenu comme argument des fonctions de demande alors qu'il est absent des fonctions de demande walrassienne. II le fait en scindant en deux étapes la procédure de maximisation de I'utilité. Patinkin (1947, p. 52), pour illustrer le problème considère un individu qui ne détient initialement ni actifs financiers, ni stock de biens. On peut, soutient-il, décrire ses choix en imaginant que, dans un premier temps, il arbitre entre loisir et revenu réel. II dispose alors d'un revenu monétaire qu'il peut répartir entre l'achat des divers biens qu'il désire. Sa demande pour un bien $j$, $y_{d, j}$, apparaît ainsi comme une fonction du prix des divers biens et de son revenu monétaire. Elle est homogène de degré zéro dans l'ensemble de ses arguments. La fonction de dépenses agrégée, $Y_{d}=\sum_{j} p_{j} y_{d, j}$, est homogène de degré 1 dans les prix et le revenu monétaire, c'est-à-dire qu'une hausse de $10 \%$ du niveau général des prix et du revenu monétaire accroît de $10 \%$ la dépense agrégée. Notons $p$ un indice des prix, on peut écrire que la demande globale réelle de biens est une fonction du revenu réel, $y$ :

$$
Y_{d}=Y_{d}(p, Y) \quad \Rightarrow \quad y_{d} \equiv \frac{Y_{d}}{p}=y_{d}(y)
$$

Sur cette base, Patinkin (Ibid., p. 64) écrit un modèle macroéconomique qui comprend trois types de biens : les biens proprement dit, les titres et la monnaie :

$$
\begin{aligned}
& y_{d}=y_{d}(y, r) \\
& y_{s}=\bar{y} \\
& y_{d}=y_{s} \\
& y=y_{s} \\
& B(r, p, y)=0 \\
& M(r, p, y)=0
\end{aligned}
$$

La première équation stipule que la demande globale de biens est une fonction du revenu réel et du taux d'intérêt. La seconde indique que l'offre globale est exogène. On pourrait aussi bien supposer que l'équilibre du marché du travail détermine le niveau de l'emploi et l'offre globale de bien. La troisième indique que le marché des biens est en équilibre. La quatrième stipule que le revenu est

\footnotetext{
12 Goulven Rubin (2002 a et b ; 2012) propose une interprétation d'ensemble de la thèse de Patinkin que, pour l'essentiel, nous partageons.
} 
égal au produit. La cinquième décrit l'équilibre sur le marché des titres. La dernière décrit l'équilibre sur le marché de la monnaie. La loi de Walras stipule qu'une de ces équations est redondante et Patinkin s'appuie sur elle pour ignorer le marché des titres. II entreprend alors d'expliquer que ce système peut être "incohérent ». Les quatre premières équations déterminent $y, y_{s}, y_{d}$ et $r$. Cependant la dernière équation pose implicitement une condition sur les valeurs acceptables du taux d'intérêt :

«Supposons que le taux d'intérêt soit négatif. Alors, puisque nous supposons que le coût de détention du papier monnaie est négligeable, tout le monde demande de la monnaie pour percevoir cet intérêt. Ainsi la demande de monnaie devient infinie. En supposant que l'offre de monnaie est finie, la demande excédentaire de monnaie tend vers l'infini. Ainsi, quand le taux d'intérêt est négatif, la sixième équation ne peut être satisfaite » (Ibid., p. 65).

Patinkin conclut de cette possibilité d'une absence de solution que le système est incohérent et doit donc être reformulé, ce qu'il fera en introduisant, en 1948, dans son article "Price flexibility and full employment" un effet d'encaisse réelle.

Pour notre objet, le point intéressant est que la réintroduction de la notion de revenu réel dans le modèle keynésien permet de discuter la question de l'existence de l'équilibre, alors que, dans la formulation de Hicks, l'existence d'un équilibre est assurée puisque la demande réelle de biens tend vers l'infini quand le prix tend vers zéro.

\section{Le choix de l'étalon et ses conséquences}

Keynes et ses successeurs ont longtemps hésité sur le choix de l'étalon dans lequel les agrégats macroéconomiques doivent être mesurés. Fallait-il choisir pour étalon la monnaie, le travail ou une marchandise composite ? La première question que l'on se posera est de se demander si en optant pour tel ou tel étalon on commet une erreur ou si ce choix est une pure question de commodité. La seconde question que l'on discutera sera celle des conséquences de ce choix.

\subsection{Où est l'erreur, s'il y en a vraiment une?}

Pour traiter de cette question, il faut d'abord commettre un crime. On contera l'histoire à rebours, en partant du choix que la profession a finalement fait, disons du modèle que Patinkin avait formulé dans sa thèse et que l'on vient de rappeler, ou, ce qui revient ici au même, du modèle que Modigliani publiera en 1963. Les solutions qui y sont retenues seront notre critère de vérité : la question sera donc de savoir si les autres solutions proposées sont équivalentes à celle-là. Les 
fonctions de demande sont des fonctions de demande réelle et on y introduit le produit, ou le revenu réel défini comme la valeur du produit divisé par un indice de prix d'une marchandise composite ou, ce qui revient au même, comme la valeur du produit à prix constant. En notant $P$ le vecteur des prix et $Q$ le vecteur des quantités produites, on aura

$$
y=\frac{P Q}{\frac{P Q}{P_{0} Q}}=P_{0} Q
$$

Adam Smith, Keynes et Lange - entre d'autres - préféraient diviser la valeur du produit par un taux de salaire, disons par le taux de salaire perçu par un travailleur non qualifié. Ils obtenaient, ainsi, la quantité de travail que commande le revenu, $\tilde{Y}$. Ce revenu est un revenu réel et le modèle obtenu ainsi est équivalent au modèle de Patinkin (1947). Modigliani utilisera cette propriété dans son article de 1963, passant sans problème d'une formulation en termes de quantités de produit à une formulation en termes de travail commandé.

On peut illustrer cette proposition en reprenant le système d'équations (2) sur lequel raisonne Lange (1938) dans son article. Si l'on suppose, comme l'a fait Lange (1938, p. 13), que le rapport du prix de chaque bien au prix du travail est donné, on passe simplement d'une écriture en travail commandé à une écriture en « volume » en multipliant $\frac{M}{W}, \tilde{Y}, \tilde{C}$ et $\tilde{I}$ par le taux de salaire réel $W / p$ ce qui est, évidemment, licite puisque ce terme est une constante :

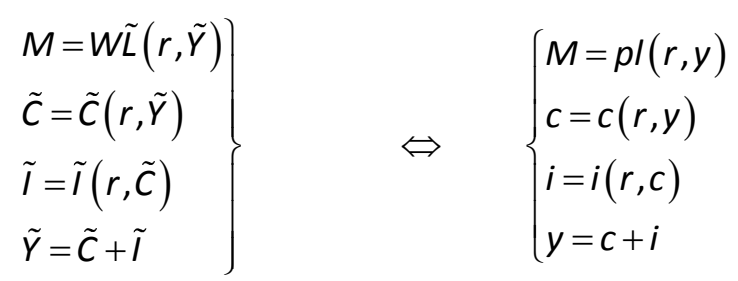

Si le taux de salaire réel est variable, le raisonnement est à peine plus complexe mais il faut introduire des hypothèses. Supposons que le produit réel soit une fonction continue strictement croissante de l'emploi : $y=f(n)$. Admettons que le salaire réel soit égal à la productivité marginale du travail, $\frac{W}{p}=f^{\prime}(n)$, alors $\tilde{Y}=\frac{y p}{W}=\frac{y}{f^{\prime}\left[f^{-1}(y)\right]}$ et l'on a 


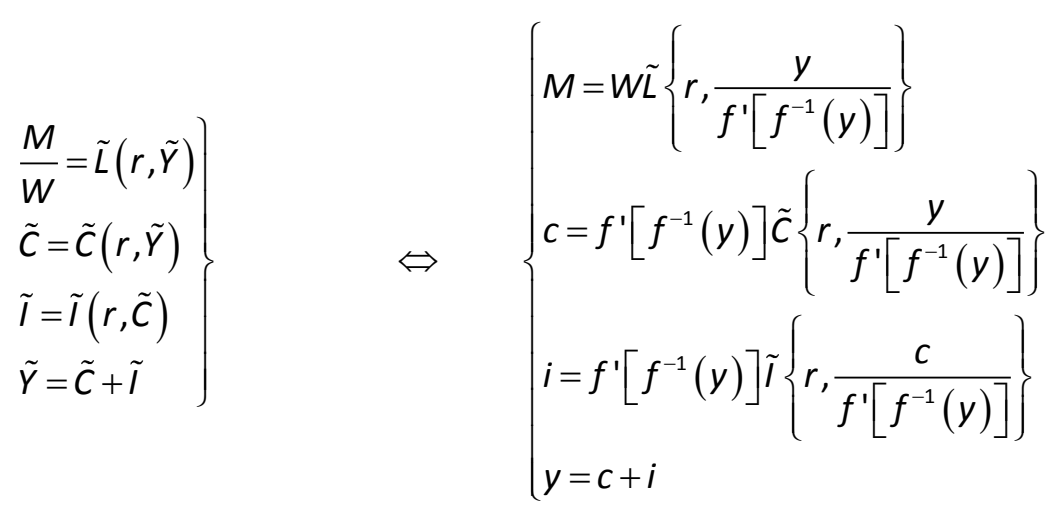

II n'est nullement nécessaire pour passer du modèle en travail commandé au modèle en volume de supposer que les fonctions de comportement sont homogènes de degré un dans le revenu. Comme le souligne Lange (1938, p. 12), peu importe le numéraire, son choix est une pure question de commodité et n'affecte ni la cohérence logique de l'analyse ni les conclusions que l'on peut en tirer. L'avantage d'une mesure en termes d'unités de salaire est illustré par les graphiques de Modigliani (1963, p. 89 et 94) et par l'usage que Keynes en fait dans la Théorie générale. Si I'on trace I'un des graphiques que Keynes a oubliés de dessiner, on voit directement comment l'offre et la demande globales déterminent le niveau de l'emploi. La Figure 4 illustre cette proposition. Les valeurs de l'offre et de la demande globales mesurées en unités de salaire sont des fonctions croissantes de l'emploi. La position et la pente de la fonction d'offre globale sont déterminées par des considérations techniques. La position de la fonction de demande globale dépend de l'offre de monnaie mesurée en unités de salaire. Quand elle augmente, la demande globale augmente. L'intersection des deux courbes détermine le niveau d'équilibre de l'emploi. La baisse du taux de salaire déplace vers le haut la fonction de demande et accroît le niveau de l'emploi. Cependant il n'existe pas nécessairement un niveau du taux de salaire qui assure le plein emploi en raison de la contrainte de positivité du taux d'intérêt. 


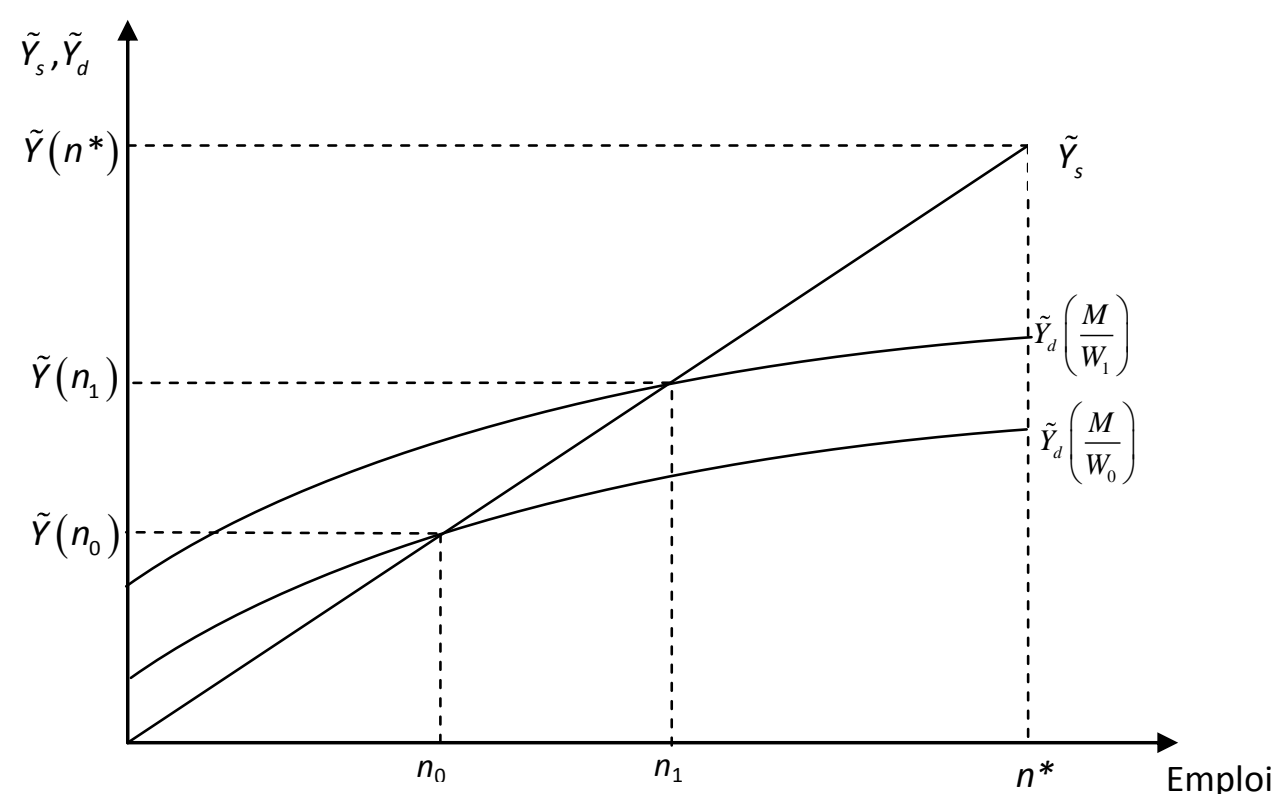

Figure 4 : L'effet d'une baisse des salaires $\left(W_{1}<W_{0}\right)$ sur l'emploi

II faut, cependant, admettre que la lisibilité des relations peut être moindre quand on raisonne en unités de salaire. Dire que le volume de l'investissement dépend du taux d'intérêt ne revient pas à soutenir que les dépenses d'investissement mesurées en unités de salaire dépendent du taux d'intérêt. En effet, la seconde proposition implique que le volume de l'investissement dépend certes du taux d'intérêt mais est, aussi, une fonction croissante du taux de salaire réel.

Le cas d'une mesure monétaire des agrégats est plus complexe. Dans la lettre qu'il adressa à Hicks le 31 mars 1937, Keynes écrivait : « Vous considérez l'épargne comme une fonction du revenu monétaire. Ceci est tout à fait correct tant que vous supposez que les salaires sont constants. Mais après avoir levé cette hypothèse [...] il n'est plus sans danger, je pense, de considérer l'épargne comme une fonction du revenu monétaire. " (Keynes, 1971-1989, t. 14, p. 80). Keynes met ainsi l'accent sur la question centrale ${ }^{13}$. Tant que le salaire monétaire est constant, il revient au même de raisonner en travail commandé ou en valeur puisque l'on peut passer de l'un à l'autre en multipliant par une constante. Si l'on interprète le modèle IS-LM comme un modèle où le salaire monétaire est non seulement exogène mais constant, alors on peut, comme Hicks, raisonner en valeur. Mais, la lecture de son article montre les difficultés auxquelles il se heurte quand il abandonne cette hypothèse pour comparer les analyses de Wicksell et de Keynes (Hicks, 1937, p. 158). Dans ce cas, postuler que le montant des sommes épargnées ou investies dépend du revenu monétaire n'est pas

\footnotetext{
13 Ingo Barens et Volker Caspari (1999) ont montré que la réaction de Keynes à l'article de Hicks reposait sur une lecture attentive de ce texte. Mais ils ne pensent pas que, quand Keynes reproche à Hicks de traiter l'épargne comme une fonction du revenu monétaire, il soulève une question fondamentale.
} 
correct car ceci implique qu'une hausse du revenu réel a, sur le montant de l'épargne et de l'investissement, le même effet qu'une hausse des prix. La même remarque vaut pour la thèse et I'article que Modigliani publia en 1944. Le problème ici est que l'idée que le salaire monétaire est variable est au cœur de la démarche de Modigliani comme en témoigne le titre de sa thèse - The general theory of employment, interest and money under the assumptions of flexible prices and of fixed prices - dont la dernière section est consacrée à l'étude de l'effet d'une baisse des salaires sur le niveau de l'emploi et la distribution du revenu. Cette partie de la thèse de Modigliani n'a pas été publiée. Son annexe mathématique (1944 a, p. 100) montre qu'il introduit, alors, la notion de produit réel dans son analyse et qu'il s'appuie sur le modèle exprimé en travail commandé tel qu'il figure dans l'article d'Econometrica (1944 b, p. 67-68). On est même tenté de suggérer que les choix que fait Keynes dans la Théorie Générale de tel ou tel étalon de valeur reflètent ce problème. Tant qu'il suppose que le salaire monétaire est donné, il raisonne en valeur ; mais quand il veut analyser les effets d'une variation des salaires sur l'emploi, il mesure les agrégats en unités de salaire.

\subsection{Les conséquences de l'erreur}

La façon dont Modigliani (1963, p. 82) caractérise les conséquences de son erreur met en évidence une série de résultats importants :

- Traiter la valeur de la consommation, ou de l'investissement, ou la demande d'encaisses nominales comme une fonction du revenu monétaire conduit à affirmer, à tort, qu'une variation des prix a sur ces variables le même effet d'une variation du revenu réel.

- Cette erreur conduit à considérer les quatre équations du système (1) qui décrivent l'équilibre sur le marché de la monnaie et sur le marché des biens comme un ensemble indépendant de la fonction de production et des équations qui caractérisent le marché du travail. Ainsi le taux d'intérêt, la valeur du revenu, de la consommation et de l'investissement ne dépendraient pas de la forme de la fonction de production - donc des chocs technologiques -, et de la valeur du taux de salaire monétaire. On peut montrer, au contraire, qu'une hausse du salaire monétaire accroît le taux d'intérêt, le niveau des prix, le revenu monétaire, la valeur de l'investissement et de la consommation tout en diminuant l'emploi, le revenu, la consommation et l'investissement réels.

On ajoutera que, dans le modèle de Modigliani (1944), une baisse du salaire monétaire accroît toujours l'emploi et le produit réel même dans le cas d'une trappe à liquidité. La 
conséquence, cependant, la plus remarquable est qu'il existe toujours, même en l'absence d'un effet d'encaisse réelle, un équilibre de plein emploi. On retrouve ainsi le résultat que l'on a établi dans la section 3.1 pour le modèle de Hicks. La démonstration est cependant plus simple.

Imaginons que le taux d'intérêt ne puisse diminuer en-dessous d'un niveau $r_{\min }$. Le revenu monétaire qui correspond à ce taux est déterminé par l'égalité de l'épargne et de l'investissement

$$
I\left(Y, r_{\min }\right)=S\left(Y, r_{\min }\right)
$$

Soit $Y\left(r_{\text {min }}\right)$ ce revenu monétaire. La quantité réelle de biens qui est demandée est $y_{d}=\frac{Y\left(r_{\text {min }}\right)}{P}$. Elle tend vers l'infini quand le niveau général des prix tend vers zéro et vers zéro quand le niveau général des prix tend vers l'infini. Quel que soit le niveau du produit réel $y^{*}$ qui assure le plein emploi, il existe toujours un prix positif tel que l'offre de biens soit égale à la demande.

$\mathrm{Si}$, au contraire, on admet que l'épargne et l'investissement réels sont fonctions du revenu réel, l'égalité de l'épargne et de l'investissement détermine pour un taux d'intérêt $r_{\min }$, un revenu réel $y\left(r_{\min }\right)$. La quantité réelle de biens qui est demandée est inférieure ou égale à $y\left(r_{\text {min }}\right)$. Si le produit réel qui assure le plein emploi excède $y\left(r_{\text {min }}\right)$, il n'existe pas d'équilibre de plein emploi. On retrouve ainsi l'idée que Keynes (1936, p. 218) avait avancée dans La Théorie Générale.

\section{Conclusion}

L'analyse de la question du choix de l'unité de mesure dans les modèles de type IS-LM suggère trois remarques. On a souvent discuté de la nature des rapports qui existent entre les modèles IS-LM et l'œuvre de Keynes. De la lecture de la lettre du 31 mars 1937 que Keynes adressa à Hicks après avoir lu son article, on a tiré des conclusions opposées. Patinkin (1990, p. 214) y voit la preuve d'une approbation qui n'est certes pas sans réserves mais qui reste notable. Pour justifier cette opinion, il cite, en particulier, la phrase où Keynes écrit "qu'il a trouvé [l'article] très intéressant et qu'il n'a presque rien à dire en matière de critique » (Keynes, 1973, t. 14, p. 79). Darity et Young $(1995$, p. 12) pensent que cette phrase est une simple formule de politesse et que Keynes est, en fait, très critique. La publication des notes des étudiants qui assistaient aux cours que donnait Keynes permet d'établir clairement (Dimand, 2007) que Keynes leur enseignait une version de ce qui allait devenir le modèle IS-LM. Plusieurs des articles qui furent rédigés après la publication de la Théorie Générale furent l'œuvre d'anciens étudiants de Keynes qui, bien sûr, tirèrent profit de son enseignement. C'est ainsi que furent conçues les idées sur lesquelles s'appuie le modèle IS-LM. 
La question du choix de l'étalon de mesure laissa perplexe aussi bien les étudiants de Keynes que, plus généralement, ses lecteurs. Tous notèrent son rejet de la notion de revenu réel ; mais ils hésitèrent quand ils eurent à choisir une solution de remplacement. Certains mesurèrent les agrégats en monnaie, d'autres en quantités de travail commandé, d'autres en quantités de travail effectué. Cependant, la plupart, notamment Hicks, raisonnèrent sur des grandeurs exprimées en monnaie. On a montré que Keynes avait raison quand il écrivait à Hicks que le choix de cette unité de mesure n'est acceptable que si le salaire monétaire est fixe ${ }^{14}$. Raisonner en valeur a deux conséquences malencontreuses. Le modèle est dichotomique et ne permet donc pas d'analyser rigoureusement les interactions entre le marché du travail d'une part et les marchés des biens et de la monnaie d'autre part. En particulier, il ne permet pas de prendre en compte les effets d'une variation du taux de salaire monétaire sur le taux d'intérêt et le revenu monétaire. Il laisse à penser qu'il existe toujours un équilibre de plein emploi pour peu que le salaire monétaire soit flexible. Pour traiter de l'existence de l'équilibre, pour étudier les rapports entre le marché du travail et les autres marchés, pour expliquer les effets d'une variation du taux de salaire monétaire, il faut raisonner sur des grandeurs réelles, c'est-à-dire choisir un numéraire. Peu importe lequel - ce peut être un panier de biens ou le travail ou une marchandise quelconque - le choix est une pure question de commodité.

On a longtemps considéré que le modèle IS-LM appartenait à la tradition walrassienne, peutêtre parce qu'il mettait l'accent sur l'interdépendance des marchés, peut-être en raison du rôle que joua Hicks dans la diffusion des idées de Pareto et de Walras parmi les économistes anglophones. De Vroey (1999, p. 118) soutient contre Patinkin (1987) et Clower (1975) que le modèle IS-LM appartient à la tradition marshallienne. Ce que l'on observe ici, c'est une série complexe d'interactions. Keynes est, de par sa formation, un marshallien; mais, sur bien des points, il s'écarte de la tradition. Par exemple, quand il discute, dans la Théorie Générale, du choix des unités, il critique la définition que Marshall et Pigou proposaient du dividende national, comme d'un revenu réel et non comme d'un revenu monétaire. Mais son effort essentiel porte sur l'analyse de l'interdépendance des marchés. Cependant, il peine à expliquer les relations entre le marché du travail et les autres marchés. C'est sur cette même difficulté que buteront, comme on l'a vu, Hicks et Modigliani. C'est à travers les contributions de Lange et de Patinkin que l'influence walrassienne se fera sentir et que les propriétés des fonctions d'offre et de demande qui découlent de l'écriture de la contrainte budgétaire seront mises en évidence. Mais, là encore, ce n'est pas sans difficultés. La façon dont Patinkin, dans sa thèse, analyse les choix des agents témoigne de ses hésitations. L'idée que les agents

14 II y a là matière à l'étonnement. Le message de Keynes dans ses cours et dans la Théorie Générale a été perçu comme ambigu par ses étudiants et par ses lecteurs. Les interprétations qu'ils en firent sont différentes. Pourtant, le texte de la lettre que Keynes adressa à Hicks laisse à penser qu'il avait correctement résolu le problème. 
détermineraient, d'abord, leur offre de services puis, compte-tenu de leurs revenus, leur demande de biens apparaît, pour un walrassien, comme curieuse. Elle évoque le souvenir de Marshall, de la façon dont il concevait les échanges comme un processus séquentiel où les facteurs sont échangés avant les produits finals ${ }^{15}$. L'histoire suggère que le modèle IS-LM initialement conçu à partir d'une approche marshallienne évolue progressivement quand des économistes, comme Lange et Patinkin, y introduisent des éléments tirés de la tradition walrassienne.

\section{Références}

BARENS, I. (1999), "From Keynes to Hicks - an aberration? IS-LM and the analytical nucleus of the General Theory", in P. Howitt, E. de Antoni and A. Leijonhufvud (eds.), Money, Market and Method. Essays in Honour of Robert W. Clower, Cheltenham and Northampton, Edward Elgar, pp. 85-120.

BARENS, I. and V CASPARI (1999), "Old Views and New Perspectives", European Journal of the History of Economic Though, vol. 6 n², pp. 216-241.

Champernowne, D. G. (1936), "Unemployment, Basic and Monetary: The Classical Analysis and the Keynesian", Review of Economic Studies, vol.3 n³, pp. 201-16.

CloWER, R. W. (1975), "Reflections on Keynesian Perplex", Zeitschrift für Nationalökonomie, vol. 35, n०1-2, pp. 1-24.

DARITY, W. and W. Young (1995), "IS-LM: An Inquest", History of Political Economy, vol.27 n¹, pp. 140

De VRoey, M. (1999), "Keynes and the Marshall-Walras divide", Journal of the History of Economic Thought, vol. 21, $\mathrm{n}^{\circ} 2$, pp. 117-136.

De VRoey, M. (2000), "IS-LM à la Hicks versus IS-LM à la Modigliani”, History of Political Economy, vol.32, n², pp. 293-316.

DIMAND, R. W. (2007), "Keynes, IS-LM and the Marshallian Tradition", History of Political Economy, vol. 39, n¹, pp. 81-95.

HAYEK, F. (1931), "Reflections on the Pure Theory of Money of Mr. J. M. Keynes", Economica, n³3, pp. 270-295.

HICKS, J. R. (1937), "Mr. Keynes and the 'Classics': A Suggested Interpretation”, Econometrica, vol. 5, $\mathrm{n}^{\circ} 2$, pp. 147-159.

KEYNES, J. M. (1909), The Method of Index Numbers with Special Reference to the Measurement of General Exchange Value, in Keynes (1971-1989), vol. 11, pp. 49-156.

KEYNES, J. M. (1930), A Treatise of Money, in Keynes (1971-1989), vol. 5 and 6.

KEYNES, J. M. (1931), "The Pure Theory of Money. A Reply to Dr. Hayek", Economica, n³4, pp. 387397.

KEYNES, J. M. (1936), The General Theory of Employment, Interest and Money, in Keynes (1971-1989), vol. 7.

KEYNES, J. M. (1971-1989), The Collected Writings of John Maynard Keynes, edited by Donald Moggridge, Cambridge: Macmillan and Cambridge University Press.

KLEIN, L. R. (1947), The Keynesian Revolution, Macmillan: New York.

KoURI, P. J. K. (1986), "Franco Modigliani's Contributions to Economics", Scandinavian Journal of Economics, vol. 88, n², pp. 311-334.

LANGE, O. (1938), "The Rate of Interest and the Optimum Propensity to Consume", Economica, vol. 5, n¹7, pp. 12-32.

15 Goulven Rubin (2012) analyse la thèse de Patinkin et met, en particulier, en évidence ce point. 
LANGE, O. (1942), "Say's Law: A Restatement and Criticism", in O. Lange, F. Mclntyre et T.G. Yntema (eds.) Studies in Mathematical Economics and Econometrics in Memory of Henri Schultz, Chicago, The University of Chicago Press, pp. 49-68.

LeiJonhufvud, A. (1994), "Hicks, Keynes and Marshall", in H. Hagemann et O.F.Hamouda (eds), The Legacy of Hicks. His contribution to economic analysis, London and New York, Routledge, pp. 143-158.

Modigliani, F. (1944 a), The General Theory of Employment, Interest and Money under the Assumptions of Flexible Prices and of Fixed Prices, Thesis, Doctorate for Social Science, New School for Social Research.

ModigliaNI, F. (1944 b), "Liquidity Preference and the Theory of Interest and Money", Econometrica, vol. $12, \mathrm{n}^{\circ} 1$, pp. $45-88$.

ModigliANI, F. (1963), "The Monetary Mechanism and its Interaction with Real Phenomena", Review of Economics and Statistics, vol. 45, $\mathrm{n}^{\circ} 1$, part 2 Supplement, pp. 79-103.

PATINKIN, D. (1947), On the Consistency of Economic Models: A Theory of Involuntary Unemployment, Thesis, University of Chicago.

Patinkin, D. (1948 a), "Relative Prices, Say's Law and the Demand for Money", Econometrica, vol. 16, $\mathrm{n}^{\circ} 2$, pp. 135-154.

PAtinkin, D. (1948 b), "Price flexibility and full employment", American Economic Review, vol. 38, $\mathbf{n}^{\circ} 4$, pp 543-564.

PATINKIN, D. (1987), "Keynes, John Maynard (1883-1946)", The New Palgrave Dictionary of Economics, eds. Steven E. Durlauf and Lawrence E. Blume, Palgrave Macmillan, The New Palgrave Dictionary of Economics Online. Palgrave Macmillan. 27 April 2012. http://www.dictionaryofeconomics.com/article?id=pde2008 K000016

PATINKIN, D. (1990), "On Different Interpretation of the General Theory", Journal of Monetary Economics, vol. 26, n², pp. 205-243.

Pigou, A. C. (1943), "The Classical Stationary State”, Economic Journal, vol. 53, n²12, pp. 343-351.

ReddaWAY, W. B. (1936), "The General Theory of Employment, Interest and Money", Economic Record, vol. 12, n¹-2, pp. 28-36.

RobInson, J. (1971), Economic Heresies, Some Old-Fashioned Questions in Economic Theory, London: Macmillan, traduction française, Paris: Calmann-Levy, 1972.

RuBiN, G. (2002 a), La contribution de Don Patinkin à la 'synthèse néoclassique' : genèse et portée, Thèse de l'Université de Paris X-Nanterre.

RUBIN, G. (2002 b), "From Equilibrium to Disequilibrium: The Genesis of Don Patinkin's Interpretation of Keynesian Theory", European Journal of History of Economic Thought, vol. 9, n², pp. 205225.

RUBIN, G. (2007), "Oskar Lange and the Origins of Walrasian Macroeconomics", ESHET Conference, Strasbourg.

Rubin, G. (2012), "Don Patinkin's PhD Dissertation as the Prehistory of Disequilibrium Theories", History of Political Economy, vol. 44, n², pp. 235-276.

RYMES, T. K. (ed.) (1989), Keynes's Lectures, 1932-35, Notes of a Representative Student, Basingtoke, Macmillan.

Samuelson, P. A. (1946), "Lord Keynes and the General Theory", Econometrica, vol. 14, n³, pp. 187200.

TORTAJAdA, R., (éd.) (2009), L'économie retrouvée, Villeneuve d'Ascq : Presses Universitaires du Septentrion.

YounG, W. (1987), Interpreting Mr. Keynes: The IS-LM Enigma, Boulder, Colo.: Westview. 